\title{
Breastfeeding New Born in Side Lying Position - Reflecting Upon a Clinical Encounter
}

\author{
Sarmad Muhammad Soomar* and Khadija Shafiq Dossa \\ Aga Khan University, Karachi Pakistan \\ Received: 㘹August 06, 2018; Published: 䧳 August 10, 2018 \\ *Corresponding author: Sarmad Muhammad Soomar, Aga Khan University, Karachi Pakistan
}

\section{Editorial}

Breast feeding is essential to positively impact an infant's health, nutrition, survival and growth. It is the great foundation of nourishment for an infant [1]. There are number of positions to facilitate comfortable breastfeeding. Among these positions, side lying is a position in which a woman lies laterally with the infant lying beside her. It is considered as most convenient position because it is not only fulfilling infant's demand of being fed but it also decreases maternal discomfort after the birth. The position itself has benefits but on the same side it has risks associated with it, which should be considered while counseling mother regarding breastfeeding. In reproductive health practice at a secondary hospital of Pakistan, we were able to identify the described situation and learn few insights from the situation, when we observe and meet a 23 years old Primigravida. She was admitted at 38th week of gestation for a delivery via Caesarean section.

On her second post-operative day, we observed her, when she was feeding her 2 days old daughter in a side lying position. Watching her doing this, we promptly approached the mother and asked her to feed the baby in a proper football position while cradling the baby in her arms. As we knew that there are certain risks associated with side lying positions which might affect the new born. It is important for mothers especially the new ones, to avoid negative outcomes for the new born. Hearing that her breastfeeding technique is improper, she became very anxious. Moreover, she told us that this is her first baby and she has seen her elders using side lying position and she practice this in order of following them. We assured her by a discussion that it's common for a primigravida to face difficulties because of a totally new experience and it is important to ask questions whenever difficulty is felt. Additionally, we told her some other breast feeding positions e.g. cradle and football positions with proper upright burping techniques to facilitate proper breastfeeding in future. Also, we describe her in detail that knowing this information and practicing it can prevent associated risks to new born's health. She was relieved from her fear and was happy with the discussion that we did.
As this was also our new experience of being a reproductive health nurse, we were initially I feeling nervous and uncertain. Beside that a doubt about my knowledge which was contrary to the client practice left us in a huge confusion. However, talking to the mother (client) and dealing her concerns made us feel helpful and improved our confidence. Moreover, the prompt change into mother's behavior (by immediately holding the new born in football position) boosted us as new reproductive health nurses and made us trust our knowledge, learning and communication. Reviewing the experience later in literature, we knew that among all breastfeeding positions, side lying, is the most preferable. This is because it allows the mother to have required rest and facilitates to relieve postpartum fatigue. Despite of its numerous benefits there are certain risks associated with it such as, it can increase the risk of mother falling asleep and potentially suffocating the baby [2]. Moreover, the baby may find difficulties during sucking and ingestion of milk which can lead to aspiration of breast milk and may experience sudden infant death syndrome [3]. As we were well aware about this concept, so we succeeded to inform the mother (client) as well.

Feldman and Whyte [4] also believe that side lying breastfeeding position is a risk factor for postnatal neonatal death. Therefore, educating mothers and their families regarding the potential dangers of feeding their babies in such positions is highly important. They further suggest that it is the responsibility of health care professionals to closely observe mothers during breastfeeding to prevent neonatal deaths due to such practices. Reflecting back to the incident, we realize that although we successfully identified one of the deficits in mother's knowledge, but others remained neglected. Ejaz and Ahmad [5] highlights that primigravida face many self and child care related issues during their postpartum period. As our client was a new mother too, it same goes for her. We in depth conducted an interview of her and identified the teaching needs that were addressed later. Apart from this, we involved other nurses those were assigned in the particular setting so that they 
can facilitate her in our absence and provide her with more specific knowledge.

In future, if we will encounter the same situation, we will try to act and provide care to clients in similar way and would like to work on their misconceptions and building their knowledge on scientific based evidences. Also, we will try to inculcate the nursing knowledge and theories appropriately to facilitate and support clients' need. This experience also provided us with a learning that we must consider bringing different teaching methodologies like video clips, copy of relevant literature and pamphlets for client's better understanding. Furthermore, we can work on developing standard plans that are always followed by nurses whenever we leave or new staff join. These must be an outcome of a literature study and experiences from evidence based learnings. Also, we will involve faculty of nursing and leadership (like head nurse, clinical nurse instructor etc.) to involve with us in developing these models and standard to practice in their particular setting. After these pilots are tested we can further more work on nurses' training and evaluation so that they can practice these suggestions. It is clear therefore, that breastfeeding in side lying position can pose risks to a newborns life. These risks may include, mother overlying during feeding, suffocation, aspiration of breast milk and sudden infant death syndrome (SIDS). Therefore, it is our professional duty to properly counsel the mothers regarding potential risks associated with such positions and provide them with alternatives. Also, as a nurse it is our responsibility and obligation connect our theoretical knowledge into practice and allow infants to undergo safe breastfeeding. Lastly, the experience helped us in reflecting many paradigms of care and that can be utilized in future practice.

\section{References}

1. United Nations Children's Fund [UNICEF] (2008) The State of the World's Children 2008 New York: UNICEF, USA p. 11-13.

2. Buswell S, Spatz D (2007) Parent-infant co-sleeping and its relationship to breastfeeding. Journal of Pediatric Health Care 21(1): 22-28.

3. Ramirez T, Malloy M (2013) Sudden infant death syndrome: are we any closer to identifying which infants will be affected? Pediatric Health, Medicine and Therapeutics 4: 13-21.

4. Feldman K, Whyte R (2013) Two Cases of Apparent Suffocation of Newborns During Side-lying Breastfeeding. Nursing for Women's Health 17(4): 337-341.

5. Ejaz S, Ahmad K (2013) Postpartum Care Utilization among Primigravida: A Study in Rural Punjab, Pakistan. Research on Humanities and Social Sciences 3(4): 10-19.

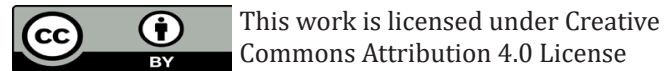

To Submit Your Article Click Here:

Submit Article

DOI: $10.32474 /$ LOJMS.2018.01.000103

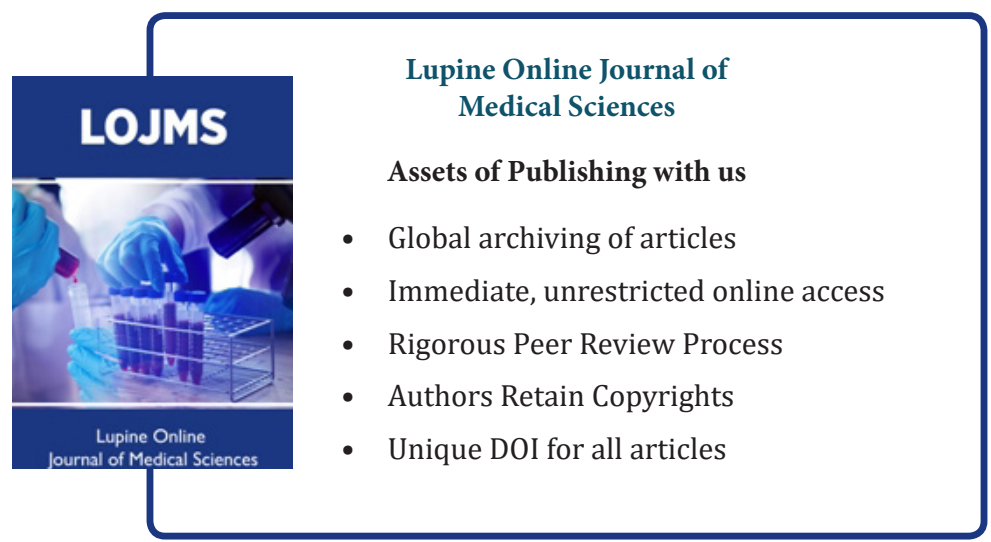

